\title{
Tipps und Tricks für die Anfertigung des perfekten Röntgenbilds im Feld - Beispiel Vordergliedmaße
}

Michael Schöberl

\author{
Aus gegebenem Anlass, der Novellierung des Röntgenleitfadens (RöLF 2018), sollen \\ im Folgenden die technischen Erfordernisse, die Vorbereitung zum Röntgen, Strah- \\ lenschutz, Bildqualität und Röntgentechnik im Kontext mit den Neuerungen be- \\ leuchtet werden und dem Praktiker Tipps und Tricks an die Hand gegeben werden.
}

\section{Vorbereitung}

Die Säuberung der Gliedmaße von Schmutz, das Ausschneiden des Hufs und das Ausfüllen des Strahls mit Knetmasse sind zur Vermeidung von Artefakten selbstverständlich. Neben den handelsüblichen Hilfsmitteln (Hufbock für Oxspringaufnahmen, Plattenhalter nach Ueltschi für Tangentialaufnahmen) sind Holzbretter gleicher Höhe zum Hochstellen der Gliedmaße notwendig.

\section{Strahlenschutz}

Neben der Qualität der Aufnahmen spielt der Strahlenschutz eine wichtige Rolle. Eine Verbesserung der Aufnahmetechnik trägt zum Strahlenschutz und zur Qualität des Röntgenbildes bei. Die Einhaltung des Strahlenschutzes ist eine unabdingbare medizinische und standespolitische Bedingung für die zukünftige ambulante Erstellung von Röntgenaufnahmen.

Durch das Anlegen passender Schutzkleidung wird die Strahlenbelastung ca. um den Faktor 30 vermindert [1]. Die Verwendung einer Bleischürze (mit ausreichendem Bleigleichwert), von Bleihandschuhen und einem Plattenhalter sind Grundvoraussetzungen. Das Tragen eines Dosimeters ist Pflicht. Näheres ist der Strahlenschutz- und Röntgenverordnung und z.B. auch den Empfehlungen des Bundesamtes für Strahlenschutz (www.bfs.de/DE/ themen/ion/strahlenschutz/beruf/beruf_node.html) zu entnehmen.

Das Röntgen findet am besten hinter einer Ziegelmauer von mindestens $12 \mathrm{~cm}$ Dicke statt. Die Sedation des Pferdes ist eine sinnvolle Maßnahme, da Verletzungen des Röntgenpersonals und des Pferdes selbst vermieden werden. Das Pferd steht ruhiger, also sinkt die Anzahl verwackelter Aufnahmen. Einblenden (Kollimation), Plattenhalter und die Benutzung eines Stativs stellen weitere Hilfsmittel dar.

\section{Bildqualität}

Eine gute Röntgenaufnahme wird bestimmt durch:

- Auflösung,

- Schärfe und

- Kontrast.

Diese Bezeichnungen sind definiert, jedoch ist das Empfinden, ob diese Werte tatsächlich erfüllt sind, subjektiv.

Ein objektiver Indikator für die korrekte Belichtung ist dagegen der relative Belichtungsindex (Relative Exposure Index). Dieser hat bei den Herstellern unterschiedliche Bezeichnungen und Empfehlungswerte:

- Rex-Zahl (Canon: 350-500)

- S-Wert (Fuji und Toshiba: 1000-2500)

Jeder Punkt des Pferdes, der von Strahlung getroffen wird, ist der Ausgangspunkt für Streustrahlung. Diese breitet sich in jede Richtung unkontrolliert aus. Die Verminderung der Streustrahlung durch Einblenden und durch das Verwenden eines Streustrahlenrasters verbessert die Qualität der Aufnahmen.

Die Positionierung des Zentralstrahls senkrecht auf den darzustellenden Bereich vermeidet eine radiografische Verzeichnung. Die Detektorplatten der digitalen Systeme und analoge Filme stellen das zu röntgende Objekt mit der besten Qualität in der Mitte der Platte dar.

Der Fokus-Film-Abstand (FFA) spielt eine wichtige Rolle für die Bildqualität. Dieser wird bei den meisten Röntgenanlagen mit $70 \mathrm{~cm}$ angegeben. Die technischen Lösungen für den korrekten Abstand reichen von Maßbändern bis zur subjektiven Einschätzung der passenden Entfernung. 


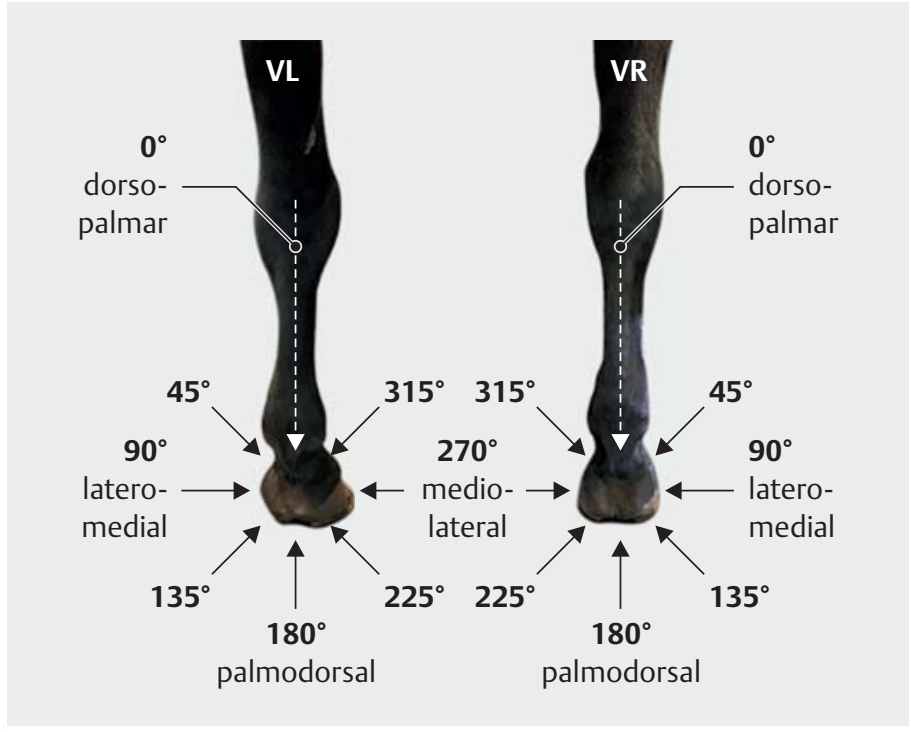

Der Autor benutzt zur Messung des FFA das Lasergerät des Röntgengeräts. Ein kurzes seitliches Zielen, neben die Gliedmaße auf die Detektorplatte, bringt die beiden Laserstreifen über Kreuz, also auf einen FFA von $70 \mathrm{~cm}$. Dies ist ein Kompromiss zwischen einer exakten Messung und der subjektiven Einschätzung des richtigen FFA.

\section{Röntgentechnik}

Eine korrekte Darstellung von Huf- und Fesselgelenk in einer Übersichtsaufnahme ist in der Regel nicht möglich, deshalb sollten Huf- und Fesselgelenk mit 2 separaten Aufnahmen dargestellt werden.

Zu den Angaben der Projektion bevorzugt der Autor das Schema nach Zeller et al. (1975) ( $\triangleright$ Abb. 1). Dieses Schema ist einfach und leicht nachvollziehbar.

- Abb. 1 Mögliche Projektionsebenen von Röntgenaufnahmen. Die Graduierung startet immer dorsokranial mit $0^{\circ}$ und schreitet nach lateral, d. h. an der linken Vordergliedmaße (VL) gegen den, an der rechten Vordergliedmaße (VR) mit dem Uhrzeigersinn, nach kaudal fort. Abbildung basiert auf Daten aus [2].

\section{Huf seitlich (lateromedial, LM, Zentrierung auf das Strahlbein)}

Zur Erstellung dieser Aufnahme empfiehlt es sich, beide Vordergliedmaßen auf Holzklötze gleicher Höhe zu stellen. Die Vorderbeine sollten unter dem Körper stehen. Die Trachten stehen im $90^{\circ}$-Winkel zur Röntgenplatte ( $\bullet$ Abb. 2). Der Zentralstrahl wird auf das Strahlbein gerichtet. Es befindet sich ca. $1 \mathrm{~cm}$ unterhalb der Hälfte des Saumbands ( $\triangleright$ Abb. 3). Eine so durchgeführte Aufnahme gewährleistet eine korrekte Beurteilung von Hufgelenk und Strahlbein ( $\bullet$ Abb. 4).

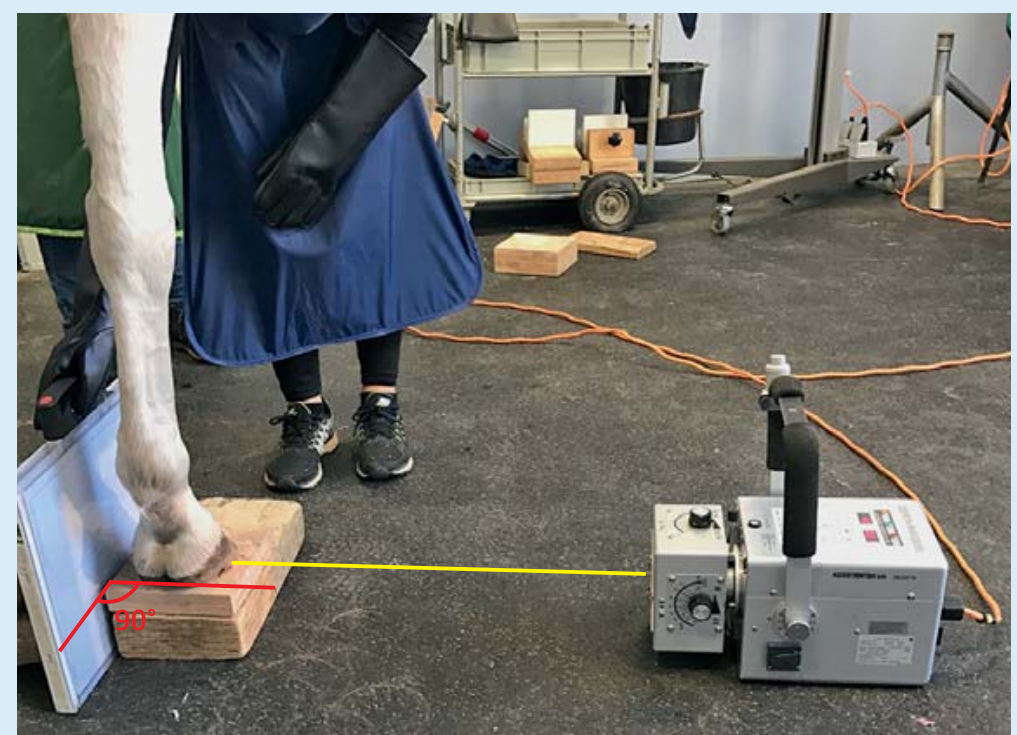

- Abb. 2 Aufnahme des Hufbeins, seitlich: beide Vordergliedmaßen stehen auf Holzklötzen gleicher Höhe. Die Vorderbeine sind unter dem Körper platziert. Die Trachten stehen im $90^{\circ}$-Winkel zur Röntgenplatte und der Zentralstrahl ist auf das Strahlbein gerichtet. (C) Michael Schöberl

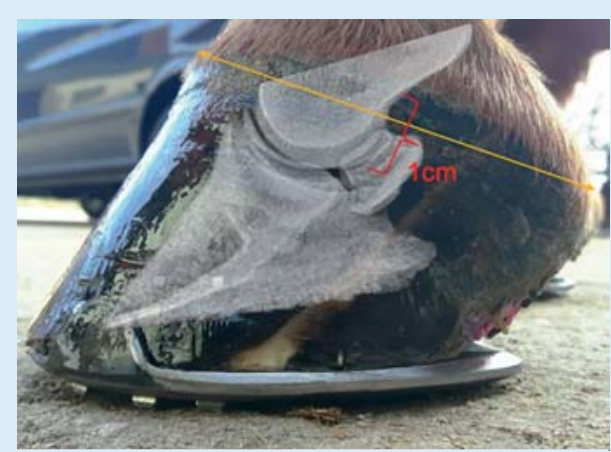

- Abb. 3 Lokalisation des Strahlbeins im Huf: es befindet sich ca. $1 \mathrm{~cm}$ unterhalb der Hälfte des Saumbands. (c) Michael Schöberl

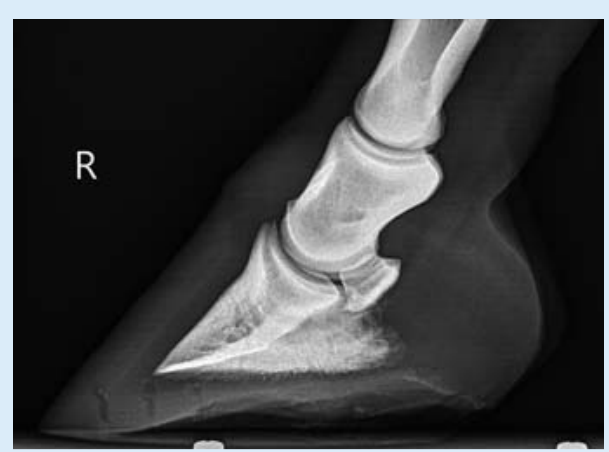

- Abb. 4 Korrekte Aufnahme des Hufgelenks: Die Hufbeinäste liegen aufeinander und die Gelenkflächen des Hufgelenks sind gut einsehbar. Das Strahlbein stellt sich als $90^{\circ}$ Schnittbild dar. (c) Michael Schöberl 


\section{Fakultative Aufnahme: $90^{\circ}$-Aufnahme der Zehe im Oxspringblock}

Die $90^{\circ}$-Aufnahme der Zehe im Oxspringblock ist eine Aufnahme, die fakultativ durchgeführt werden kann, falls fragliche Befunde vorliegen. Auch hier wird der Zentralstrahl auf das Strahlbein gerichtet. Die Detektorplatte steht parallel zur Gliedmaßenachse ( $\triangleright$ Abb. 5).

- Abb. 5 Aufnahme der Zehe seitlich $\left(90^{\circ}\right)$ im Oxspringklotz. a Seitliche Ansicht. Der Zentralstrahl wird auf das Strahlbein gerichtet. b Kaudale Ansicht. Die Detektorplatte steht parallel zur Gliedmaßenachse. (c) Michael Schöberl

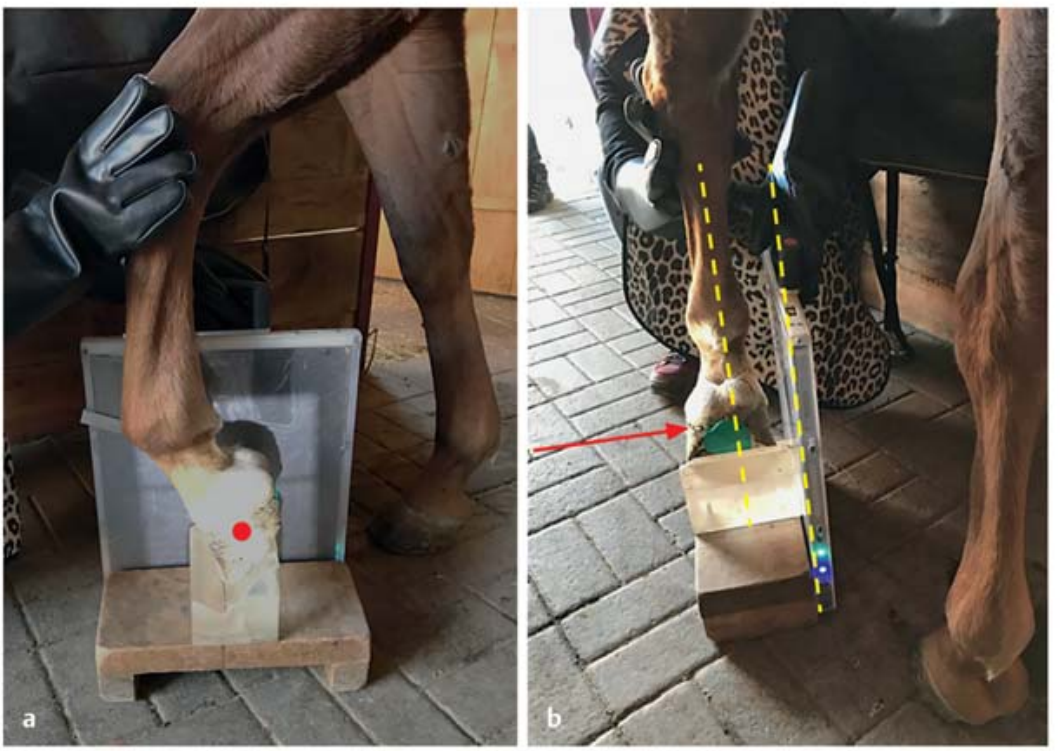

\section{Fesselgelenk seitlich (lateromedial, LM, 90 )}

Die seitliche Aufnahme des Fesselgelenks kann ohne Unterlegen eines Bretts durchgeführt werden. Beide Vorderbeine sollten gleichmäßig unter dem Körper stehen. Die Achse der Zehe ( $0^{\circ}$-Linie) sollte parallel zur Röntgenplatte verlaufen ( $\bullet$ Abb. 6). Bei einem Pferd mit korrekter Gliedmaßenstellung steht die Röntgenplatte senkrecht zum Boden. Bei einer Fehlstellung der Fessel (Varus oder Valgus) richtet sich die Stellung der Röntgenplatte nach der Gelenksachse.

Die Trachten stehen senkrecht zur Detektorplatte und der Zentralstrahl zielt auf den Gelenkspalt des Fesselgelenks. Ist die Aufnahme korrekt erstellt, liegen die beiden proximalen Gleichbeine nahezu aufeinander, der Sagittalkamm des Metacarpus III ist komplett einsehbar und die Weichteile zeichnen sich ab ( $\bullet$ Abb. 7).
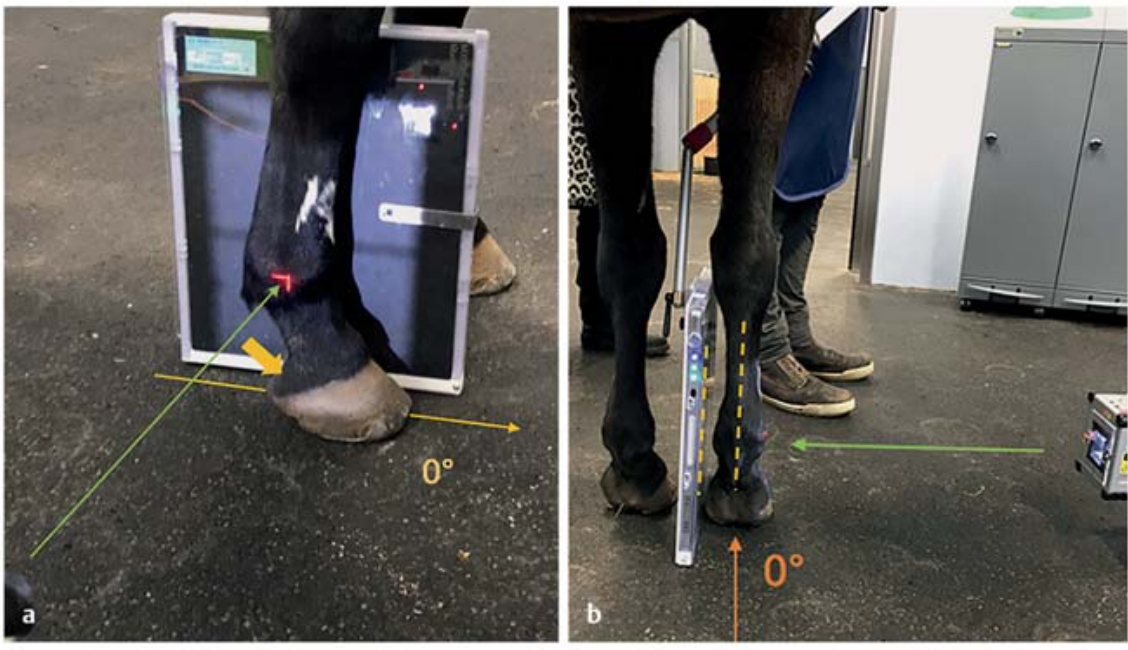

- Abb. 6 Aufnahme des Fesselgelenks, seitlich, 90: Die Aufnahme kann ohne Unterlegen eines Bretts durchgeführt werden. Beide Vorderbeine stehen gleichmäßig unter dem Körper. a Seitliche Ansicht: Die Achse der Zehe ( $0^{\circ}$-Linie) sollte parallel zur Röntgenplatte verlaufen. Der Zentralstrahl ist mittig auf das Fesselgelenk gerichtet. b Ansicht von kaudal: Bei einem Pferd mit korrekter Gliedmaßenstellung steht die Röntgenplatte senkrecht zum Boden und parallel zur Gelenksachse. Die Trachten stehen senkrecht zur Detektorplatte und der Zentralstrahl zielt auf den Gelenkspalt des Fesselgelenks. @ Michael Schöberl

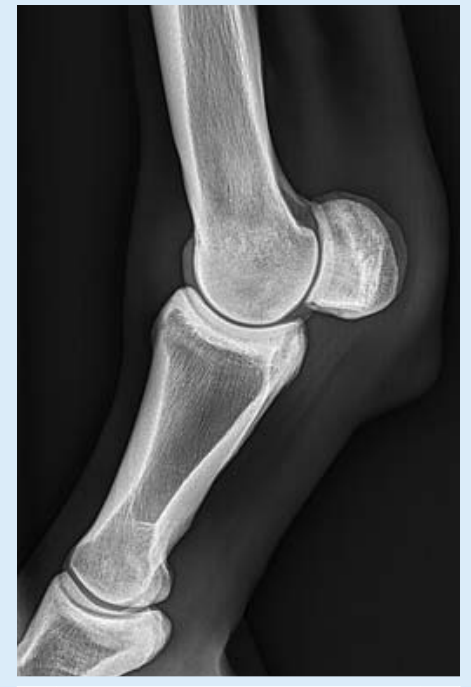

- Abb. 7 Korrekte Fesselgelenksaufnahme, seitlich, $90^{\circ}$ : die beiden proximalen Gleichbeine liegen nahezu aufeinander, der Sagittalkamm des Metacarpus III ist komplett einsehbar und die Weichteile zeichnen sich ab. (c) Michael Schöberl 


\section{Fesselgelenk (dorsopalmar, DP, $0^{\circ}$ )}

Fragliche Befunde auf der seitlichen Aufnahme und ein Frakturverdacht erfordern eine weitere Projektion der Fessel - die $0^{\circ}$-Aufnahme.

Die dorsopalmare Aufnahme der Vordergliedmaße wird durch eine weiche Fesselung erschwert, da die Gleichbeine nach distal absinken. Auf dem Röntgenbild „überlappen“ die Gleichbeine den Gelenkspalt des Fesselgelenks und machen eine Beurteilung des distalen Metacarpus und des proximalen Fesselbeins schwierig. Mittels der Aufnahmetechnik kann dies nur bis zu einem gewissen Grad ausgeglichen werden.

Das Vorderbein sollte unter dem Körper positioniert werden. Je weicher die Fesselung, desto weiter wird die Gliedmaße nach kaudal versetzt. Der Zug auf der tiefen Beugesehne führt zu einer steileren Stellung der Fessel.

Die Röntgenplatte liegt an den Ballen an und wird nach kaudal gekippt ( $\bullet$ Abb. 8).

Bei dieser Projektion ist der Einfallswinkel entscheidend, er sollte nicht senkrecht auf die Rezeptorplatte treffen, sondern etwas flacher. Der Winkel zum Boden sollte ca. $5^{\circ}$ betragen. Der Zentralstrahl ist dabei auf das Fesselgelenk gerichtet ( $\bullet$ Abb. 9).

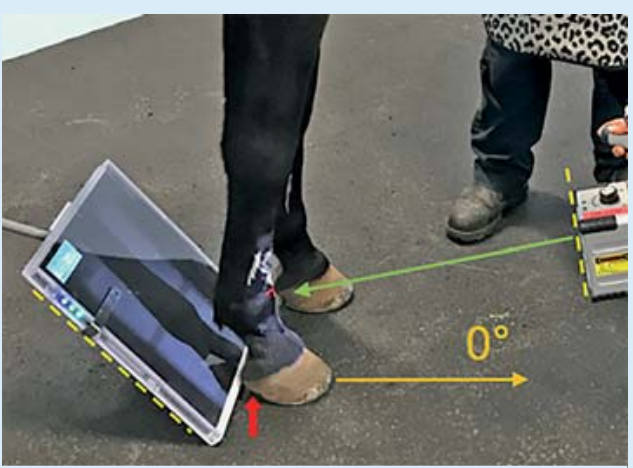

- Abb. 8 Aufnahme des Fesselgelenks, $0^{\circ}$, Einfallswinkel und Position der Röntgenplatte: Das Vorderbein steht unter dem Körper. Je weicher die Fesselung, desto weiter wird die Gliedmaße nach kaudal versetzt. Die Röntgenplatte liegt an den Ballen an und wird nach kaudal gekippt. Der Zentralstrahl ist mittig auf das Fesselgelenk gerichtet. @ Michael Schöberl

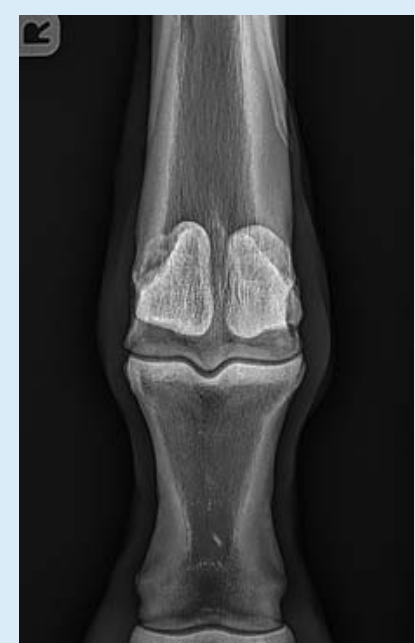

- Abb. 9 Korrekte Aufnahme des Fesselgelenks, dorsopalmar, $0^{\circ}$ : Beide Gleichbeine sind proximal des Gelenkspalts projiziert. Der distale Metacarpus, der Gelenkspalt, der subchondrale Knochen und die Weichteile sind komplett beurteilbar. (c) Michael Schöberl

\section{Aufnahme des Strahlbeins, $0^{\circ}$, nach Oxspring}

Vor der Röntgenaufnahme des Strahlbeins erfolgt die Säuberung von Sohle und Strahl mittels Hufmesser, -kratzer und Bürste. Dies vermeidet Artefakte und gilt ebenfalls für die Entfernung des Hufeisens. Das Ausfüllen der Sohle und des Strahls mit Knetmasse vermindert Streustrahlung und verhilft zu einer deutlich besseren Bildqualität.

Das Einblenden und die Benutzung eines Rasters führen zu einer deutlichen Verbesserung der Aufnahmequalität. Der Hufblock für die Oxspringaufnahme gibt den korrekten Winkel (60 $)$ für die Aufnahme vor. Die Hufsohle muss dem Block anliegen ( $\vee$ Abb. 10). Das zu röntgende Bein wird ein wenig vor den Körper platziert und die Fessel wird in Extension gedrückt.

Der Zentralstrahl geht in einem Winkel von ca. $3^{\circ}$ proximodistal und zielt etwa $1-1,5 \mathrm{~cm}$ proximal des Hufrandes mittig auf die Gliedmaße (vgl. Beispielaufnahmen ( $\bullet$ Abb. 11) der Strahlbeinaufnahme nach Oxspring.

- Abb.10 Strahlbeinaufnahme nach Oxspring. a Das zu röntgende Bein wird ein wenig vor den Körper platziert und die Fessel wird in Extension gedrückt. Die Hufsohle muss dem Block anliegen. b Der Zentralstrahl ist etwa $2-3^{\circ}$ geneigt und mittig, ca. 1-1,5 cm über den Kronrand gerichtet. (c) Michael Schöberl
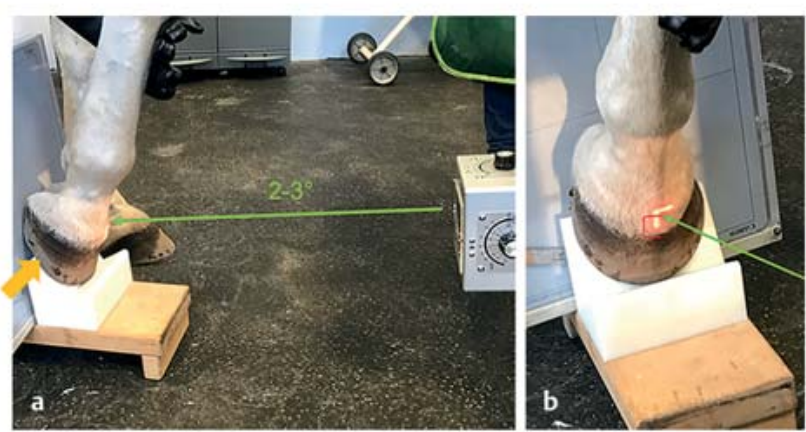

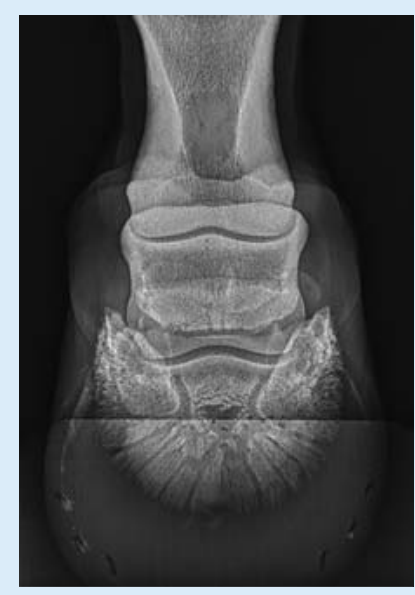

- Abb. 11 Korrekte Aufnahme des Strahlbeins nach Oxspring: Das Strahlbein ist proximal des Hufgelenksspalts dargestellt. Die Knochenstruktur, die Canales sesamoidales, die Facies articularis und flexoria sind gut beurteilbar. ( Michael Schöberl 


\section{Strahlbein,}

Tangentialaufnahme

Immer wieder weisen die klinische Untersuchung oder die „normalen“ Röntgenbilder auf ein Problem des Hufrollenapparats hin. Falls auf den Standardaufnahmen kein Befund erhoben werden kann, ist eine Tangentialaufnahme des Strahlbeins empfehlenswert. Hierzu bedarf es der gleichen Vorbereitung des Hufs wie zur Oxspringaufnahme (Eisen entfernen, Ausschneiden, Knetmasse). Bei schmerzhaften Prozessen sind eine Sedation oder eine Anästhesie der Palmarnerven erforderlich.

Die Hufform spielt für den Winkel des Zentralstrahls eine große Rolle: je steiler der Huf ist, desto stärker muss die Hyperextension des Hufgelenks sein, um das Strahlbein darzustellen. Das Stellen des ande-

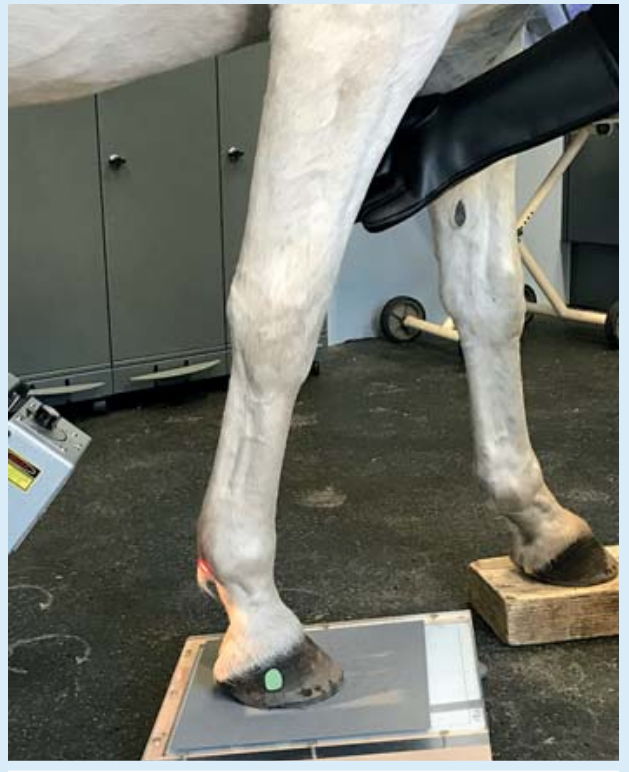

- Abb. 12 Aufnahme des Strahlbeins, Tangentialaufnahme: Das andere Vorderbein wird auf einem Holzbrett gleicher Höhe platziert. Je steiler die Hufform ist, umso stärker muss die Hyperextension des Hufgelenks sein. Die Querlinie des Zentralstrahls zielt durch das Strahlbein. @ Michael Schöberl ren Vorderbeins auf ein Holz-

brett gleicher Höhe ( $\triangleright$ Abb. 12), wie der Plattenhalter für die Tangentialaufnahme, erleichtert dem Pferd das Stillstehen. Wie in $\mathbf{A} \mathbf{A b b} \mathbf{1 3}$ ersichtlich, bewirken unterschiedliche Winkel des Zentralstrahls unterschiedliche Befunde, die falsch interpretiert werden können. Aufgrund des Einfallswinkels der Röntgenstrahlen auf die Rezeptorplatte ist die Verwendung eines Rasters nicht sinnvoll. Ein Fokusobjektabstand von $70 \mathrm{~cm}$ und die Verwendung eines Stativs sind zumeist nicht möglich. Die Querlinie des Fadenkreuzes zielt durch das Strahlbein ( $\bullet$ Abb. 12).
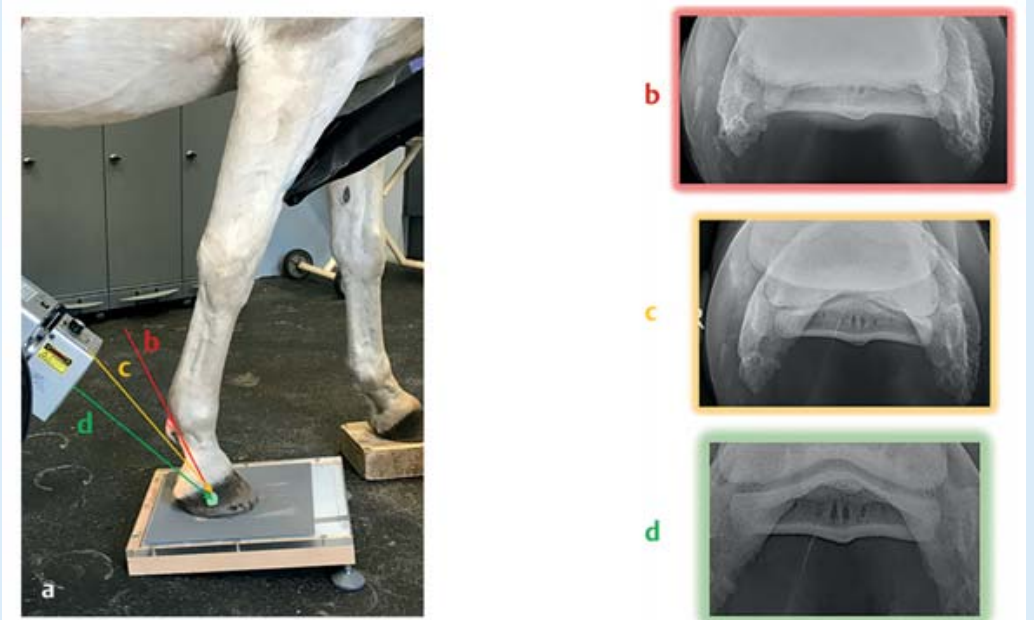

d

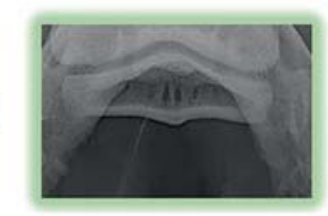

- Abb. 13 Tangentialaufnahme des Strahlbeins. a Unterschiedliche Projektionen bewirken unterschiedliche Befunde am gleichen Strahlbein. Das bedeutet, dass eine falsche Projektion zu dem Anschein einer vermehrten Sklerosierung der Spongiosa des Strahlbeins führen kann (b, c, d). Die Hufform und eine seitliche Aufnahme des Strahlbeins helfen bei der Wahl der korrekten Projektion. Faustregel: „Je flacher der Huf, desto flacher die Projektion“. (c) Michael Schöberl 


\section{Huf seitlich (lateromedial, LM) bei Hufrehe und Hufimbalance}

Die klinische Diagnose einer Hufrehe macht die Erstellung korrekter Aufnahmen des Hufs nötig. Bei dieser Röntgenaufnahme wird der Zentralstrahl nicht auf das Strahlbein, sondern auf die Sohle gerichtet. Das Abziehen des Hufeisens ist empfehlenswert. Beide Vorderbeine werden auf Holzklötze gleicher Höhe gestellt ( $\mathbf{A} \mathbf{b b}$. 14). Eine Markierung dorsal auf der Hufwand, die am Saumband („letztes Haar“) beginnt und eine Markierung an der Strahlspitze sind trotz digitaler Aufnahmetechnik notwendig ( $\triangleright$ Abb. 14). Bei Hufen, die nicht in der Balance sind, wird zusätzlich zur seitlichen Aufnahme eine $0^{\circ}$ Aufnahme des Hufes durchgeführt. Auch hier wird der Zentralstrahl auf die Sohle gerichtet. Mit diesen beiden Aufnahmen kann der Hufschmied die Imbalance beurteilen und ausgleichen.

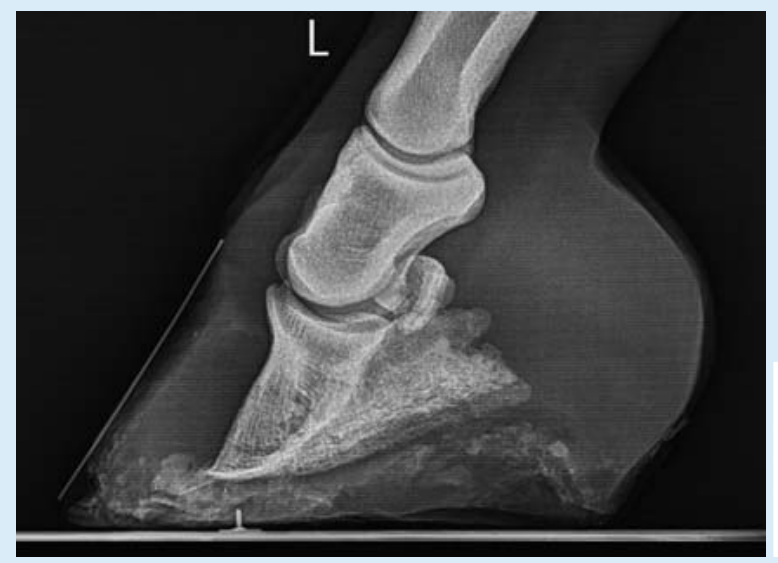

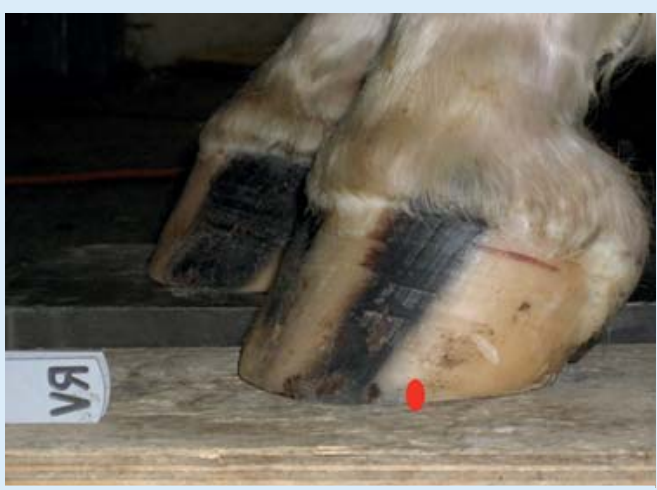

- Abb. 14 Aufnahme in der Rehediagnostik, Huf seitlich: beide Vorderbeine werden auf Holzklötze gleicher Höhe gestellt. Anbringung der Marker unterhalb des Kronrands („letztes Haar“) und an der Hufsohle vor der Strahlspitze. (c) Michael Schöberl

- Abb.15 Aufnahme in der Rehediagnostik, Huf seitlich. Bei dieser korrekten Aufnahme ist der ganze Huf dargestellt. Die Hufsohle liegt parallel zum Untergrund und ist orthograd getroffen. @ Michael Schöberl

\section{Laterales Gleichbein schräg (45-Aufnahme)}

Häufig stellt die schräge Aufnahme des Fesselgelenks und seiner Gleichbeine ein technisches Problem in der Außenpraxis dar: zumeist ist der Winkel der Projektion zu flach, wodurch die Gleichbeine auf den Gelenksspalt projiziert werden. Eine Beurteilung der Fesselbeinlehne ist so nicht möglich.

Um dies zu vermeiden, wird bei dieser Aufnahme in einem proximodistalen Winkel von ca. $15^{\circ}$ auf das laterale Gleichbein gezielt. Der Winkel der Röntgenplatte zum Boden ist senkrecht, nur bei einer sehr schlechten Gliedmaßenstellung ist die Stellung der Platte dem Vorderbein anzupassen ( $\triangleright$ Abb. 16 und 17 ).
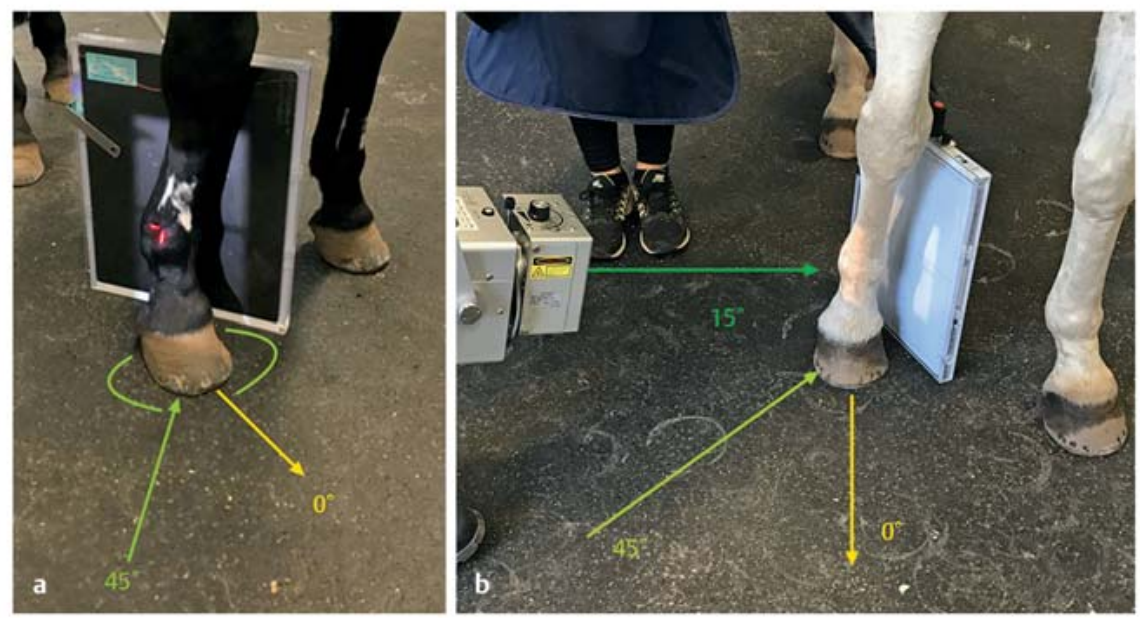

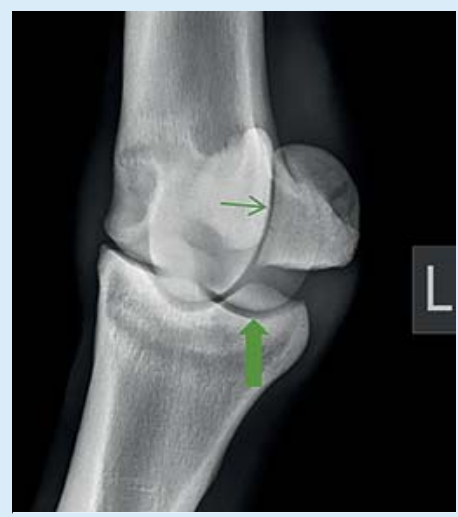

- Abb. 17 Korrekte Aufnahmen des lateralen Gleichbeins: Die Fesselbeinlehne und die Facies articularis des Gleichbeins sind komplett einsehbar. (c) Michael Schöberl

- Abb. 16 Aufnahmetechnik für das laterale Gleichbein $\left(45^{\circ}\right)$ : es wird mit einem proximodistalen Winkel von ca. $15^{\circ}$ auf das laterale Gleichbein gezielt. (c) Michael Schöberl 


\section{Mediales Gleichbein schräg}

(315-Aufnahme)

Die Aufnahme des medialen Gleichbeins erfordert die gleiche Technik wie die Aufnahme des lateralen Gleichbeins. Die Darstellung gelingt besser von medial, also $315^{\circ}$. Es ist einfacher von medial im genügend steilen Winkel $\left(15^{\circ}\right)$ auf das Fesselgelenk zu treffen ( $\bullet$ Abb. 18 und 19 ).
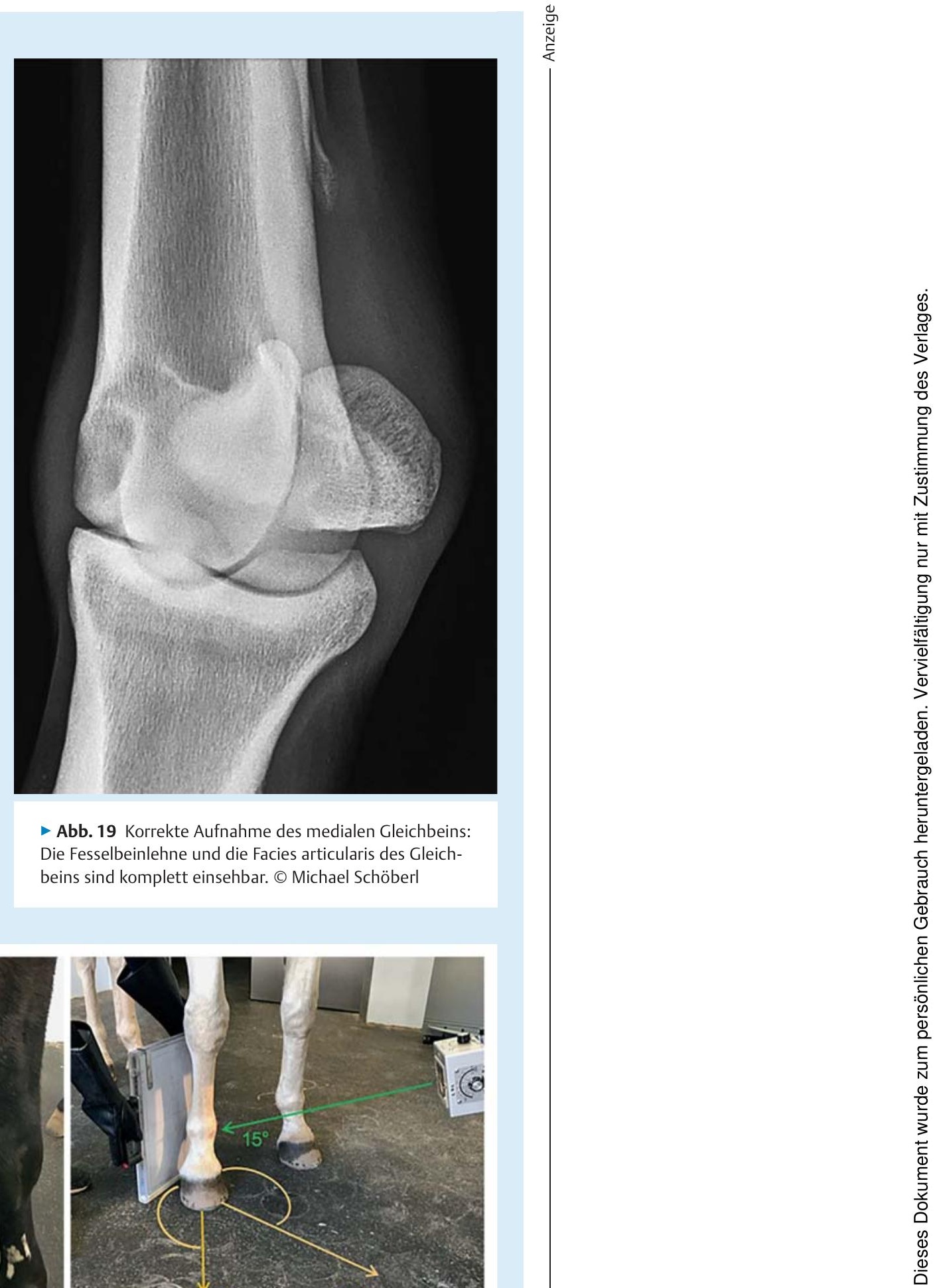

- Abb. 19 Korrekte Aufnahme des medialen Gleichbeins: Die Fesselbeinlehne und die Facies articularis des Gleichbeins sind komplett einsehbar. @ ( Michael Schöberl

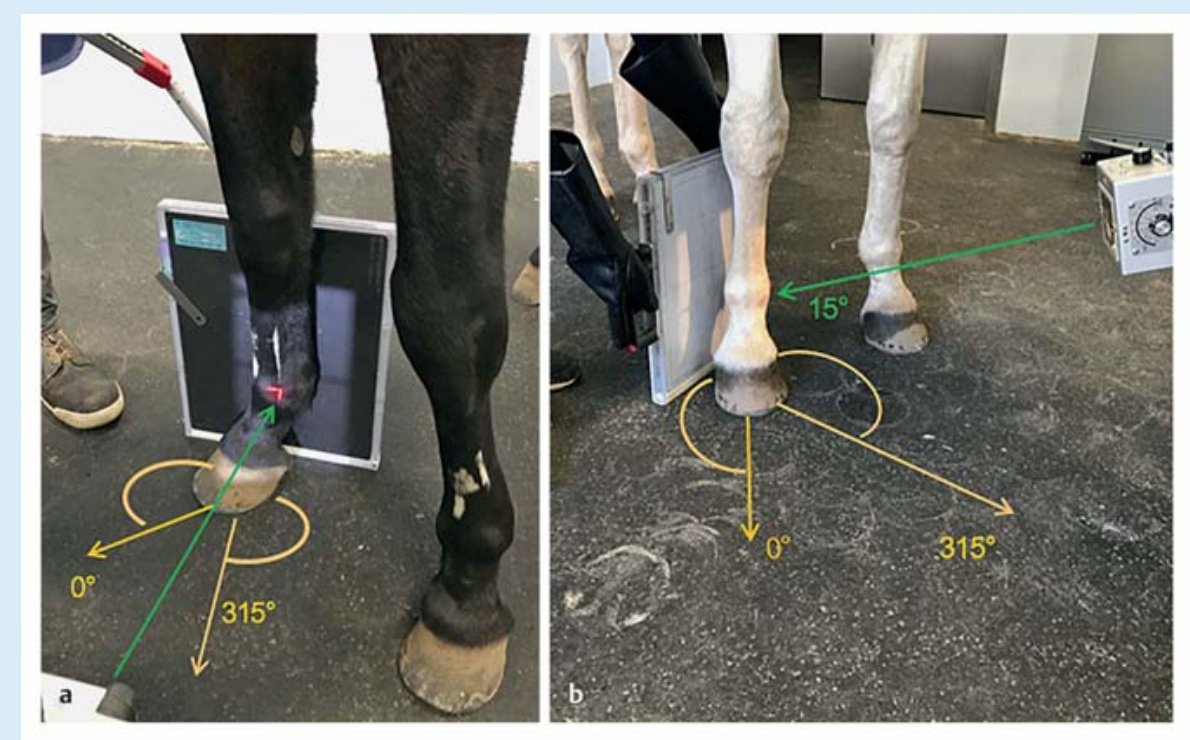

Abb. 18 Aufnahmetechnik für das mediale Gleichbein mit etwa um $15^{\circ}$ geneigter Strahlenquelle. (c) Michael Schöberl 


\section{Zusammenfassung}

1. Die digitale Röntgentechnik ermöglicht die Erstellung qualitativ hochwertiger Röntgenbilder im Feld.

2. Die Erhebung von Befunden und deren Interpretation ist ohne ausreichende Anatomiekenntnisse und korrekte Projektionen unmöglich.

3. Die Projektion und der Fokus der Aufnahme sollte sich nach der individuellen Konformation und Stellung des Pferdes richten.

4. Berücksichtigung von 2. und 3. vermindert die Strahlungsexposition.

5. Der RöLF 18 berücksichtigt dies durch:

a) technische Anweisungen

b) zusätzliche Unterteilung (Hufbein seitlich und Fessel seitlich)

c) Erhöhung der empfohlenen Anzahl der Röntgenbilder für die Ankaufuntersuchung.
Korrespondenzadresse

Dr. med. vet. Michael Schöberl

Pferdepraxis Beilngries

Bräuhausstr. 34

92339 Beilngries

michael.schoeberl@pferdepraxis.com

Literatur

[1] Tyson R, Smiley DC, Pleasant RS et al. Estimated operator exposure for hand holding portable $X$-ray units during imaging of the equine distal extremity. Vet Radiol Ultrasound 2011; 52: $121-124$

[2] Zeller R, Hertsch B, Wilkens $\mathrm{H}$ et al. Die Bezeichnung der Aufnahmerichtung bei der Röntgenuntersuchung in der Veterinärmedizin. Dtsch Tierarztl Wochenschr 1975; 82: 22-24

Bibliografie

DOI https://doi.org/10.1055/s-0044-100870

Pferdespiegel 2018; 21: 3-10

(c) Georg Thieme Verlag KG Stuttgart · New York

ISSN 1860-3203 